\title{
Semi-Automated SVG Programming via Direct Manipulation
}

\author{
Brian Hempel Ravi Chugh \\ Department of Computer Science, University of Chicago, USA \\ \{brianhempel, rchugh\} @ uchicago.edu
}

\begin{abstract}
Direct manipulation interfaces provide intuitive and interactive features to a broad range of users, but they often exhibit two limitations: the built-in features cannot possibly cover all use cases, and the internal representation of the content is not readily exposed. We believe that if direct manipulation interfaces were to (a) use general-purpose programs as the representation format, and (b) expose those programs to the user, then experts could customize these systems in powerful new ways and non-experts could enjoy some of the benefits of programmable systems.
\end{abstract}

In recent work, we presented a prototype SVG editor called SKETCH-N-SKETCH that offered a step towards this vision. In that system, the user wrote a program in a general-purpose lambda-calculus to generate a graphic design and could then directly manipulate the output to indirectly change design parameters (i.e. constant literals) in the program in real-time during the manipulation. Unfortunately, the burden of programming the desired relationships rested entirely on the user.

In this paper, we design and implement new features for SKETCH-N-SKETCH that assist in the programming process itself. Like typical direct manipulation systems, our extended SKETCH-N-SKETCH now provides GUI-based tools for drawing shapes, relating shapes to each other, and grouping shapes together. Unlike typical systems, however, each tool carries out the user's intention by transforming their general-purpose program. This novel, semi-automated programming workflow allows the user to rapidly create highlevel, reusable abstractions in the program while at the same time retaining direct manipulation capabilities. In future work, our approach may be extended with more graphic design features or realized for other application domains.

\section{Author Keywords}

Live Programming, Direct Manipulation, SVG

\section{ACM Classification Keywords}

H.5.2. Information Interfaces and Presentation (e.g. HCI): User Interfaces; D.2.6. Software Engineering: Programming Environments; D.3.3. Programming Languages: Language Constructs and Features

This is the authors' preprint version. It is posted here for your personal use. Not for redistribution. The definitive version was published in the following venue.

UIST '16 October 16-19, 2016, Tokyo, Japan (C) 2016 Copyright held by the owner/author(s).

Publication rights licensed to ACM. ISBN 978-1-4503-4189-9/16/10 ... \$15.00 DOI: http://dx.doi.org/10.1145/2984511.2984575

\section{INTRODUCTION}

Direct manipulation interfaces [33] provide a broad range of users the tools to author content in a variety of application domains. In addition to intuitive and immediate feedback, fullfeatured direct manipulation systems (e.g. Adobe Illustrator, Microsoft PowerPoint, and Apple Keynote) provide scores of built-in features such as rulers, snap-to alignment, grouping, and animations for operations common to a target domain.

Nevertheless, experts and novices alike must often resort to mundane, repetitive tasks - such as excessive copy-andpasting and secondary edits to keep conceptually related objects in sync - that could be avoided with general-purpose programming. Some direct manipulation systems provide APIs for customization, but they are typically not connected to the main application in lightweight, easy-to-use ways.

In response, researchers have recently proposed several approaches that attempt to strike a balance between intuitive interactivity and expressive programmability. We believe these prior efforts can be classified into two broad categories.

\section{Adding Programming to Direct Manipulation}

One approach has been to extend a mostly traditional direct manipulation system with "structured" programming features, by which we mean design choices that restrict the expressiveness of the language (e.g. a domain-specific language) or provide limited code editing tools (e.g. a block editor). Software tools in this category, such as Drawing Dynamic Visualizations [38], Apparatus [31], and Programming by Manipulation [17], tend to favor direct manipulation and rely on programming as a last-resort scenario.

\section{Adding Direct Manipulation to Programming}

In contrast, another approach has been to extend a mostly traditional, general-purpose programming language with direct manipulation features. Software tools in this category, such as Live Programming [40, 26, 32] and our previous version of SKETCH-N-SKETCH [9], expect that users will work closely with text-based programs but also provide ways for directly manipulating output values to indirectly edit the program.

The approaches in the first category have significant merit, especially for specific application domains. However, we favor the second approach - that general-purpose languages can be the foundation upon which to build full-featured user interfaces - because of the potential for new techniques to augment programming methodologies in general. Unfortunately, our previous effort required users to carry out the lion's share of the design work programmatically; only then could the user directly manipulate the output to indirectly modify design parameters (i.e. constants) in the program [9]. 


\section{Our Approach and Contributions}

We present new direct manipulation features for the SKETCH-N-SKETCH Scalable Vector Graphics (SVG) editor that interactively assists the user in building a program in a high-level, general-purpose programming language.

Specifically, our paper makes the following contributions:

- We design novel direct manipulation features for (1) drawing new shapes, (2) relating features among shapes, and (3) building reusable abstractions from groups of shapes. Each of the tools in our Draw, Relate, and Group workflow is paired with a program transformation that automates some of the mundane parts of programming, significantly reducing the programming burden.

- We implement our design in the context of the SKETCH-N-SKETCH system. Our implementation is opensource and publicly available on the Web.

- We demonstrate how our new SKETCH-N-SKETCH implementation can be used to author several graphic designs where the bulk of the programming is done automatically by the system. Videos of some of these examples are available on the Web. ${ }^{1}$

As a result, the user now starts by drawing and interacting with shapes on the canvas and receives guidance from the system in turning the desired relationships into a high-level, readable program, which then enables the interaction capabilities developed in our previous work for manipulating design parameters via direct manipulation.

We believe that our ideas serve as another step towards the long-term goal of truly achieving a harmonious combination of programming and direct manipulation.

\section{OVERVIEW}

We now provide an overview of how our extensions to SKETCH-N-SKETCH interactively help the user build a program that implements a reusable graphic design. The workflow in SKETCH-N-SKETCH can be viewed as a series of three phases (Drawing Shapes, Relating Features, and Grouping Shapes) which, in practice, may overlap and be interleaved. In this section, we will explain each phase in terms of a running example. Then, in three subsequent sections, we discuss each phase in more detail.

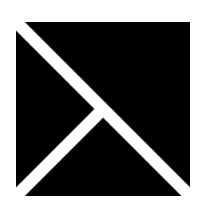

Our overview example is to build the SKETCH-N-SKETCH logo, shown on the left, which consists of three triangular positive areas separated by two equal-width negative stripes. We would like to implement this logo in a way that makes the design parameters (colors, width, and size) easy to change. Starting from an initially empty program that does not draw any shapes, we will be able to create the final program entirely using the direct manipulation tools in SKETCH-N-SKETCH. In the Evaluation section, we will describe examples that require some edits to the source code.

\footnotetext{
${ }^{1}$ http://ravichugh.github.io/sketch-n-sketch
}

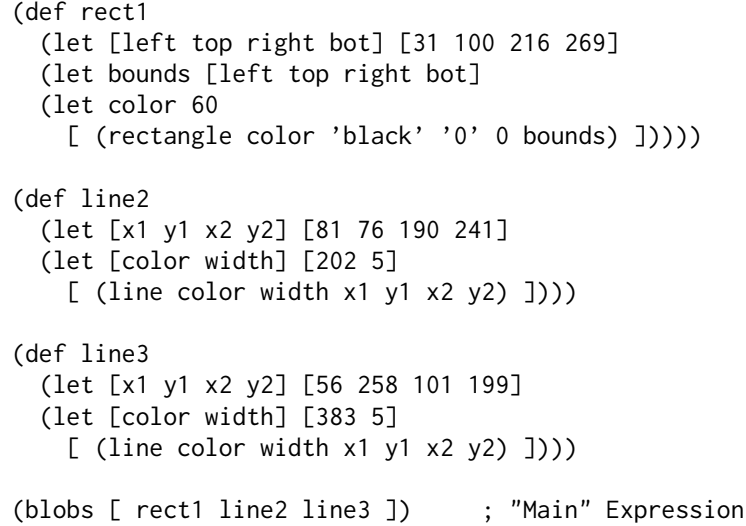

Figure 1. Overview example after drawing new shapes.

\section{Drawing Shapes}

The programming language in SKETCH-N-SKETCH, called little, is a general-purpose, untyped lambda-calculus, and the values produced by a little program are translated to the SVG format for rendering [9]. We extend SKETCH-N-SKETCH with direct manipulation drawing tools for several common shapes, so that the user does not have to write little code to add new shapes. When a shape is drawn in the canvas, the editor adds a corresponding definition to the little program such that, when re-evaluated, the program produces the new shape.

In order to implement the logo, our first step is to pick an underlying representation for the design. One option is to use a single rectangle in the background and two lines in the foreground. When we draw a rectangle and two lines very roughly positioned on top of it (as shown on the right), SKETCH-N-SKETCH generates the program in Figure 1.

The program structure comprises a series of top-level definitions followed by a "main" expression that defines the output SVG canvas. Notice that the formatting and identifier names are designed to be easy for the user to read and edit. The library functions rectangle and line draw the corresponding SVG primitives, and the blobs function denotes list concatenation. We will describe the program in Figure 1 in more detail in the Tools for Drawing Shapes section.

\section{Live Synchronization: Direct Manipulation and Widgets}

The original version of

SKETCH-N-SKETCH [9]

allowed the user to manipulate attributes in the output, causing the system to immediately infer

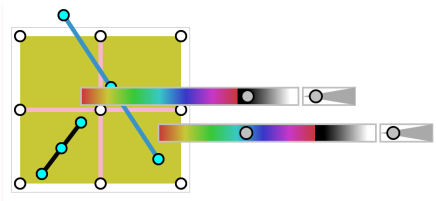
changes to constants in

the program during the user action. The size and position of shapes could be adjusted directly. Attributes without natural visual representations, such as color and line width, could be manipulated via helper widgets (the sliders in the image). We inherit this functionality without significant changes. 


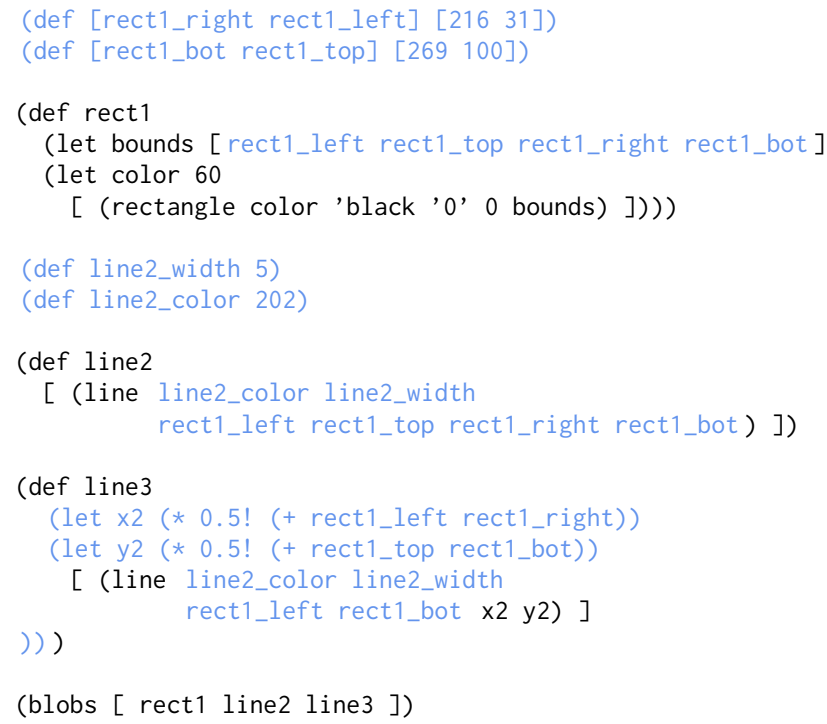

Figure 2. Overview example after relating features. The changes compared to Figure 1, made automatically by SKETCH-N-SKETCH, are highlighted in bolded blue.

\section{Relating Features}

Once the basic shapes have been defined, the next step is to introduce more structure into the program to define relationships. To facilitate this process, the Relate phase in SKETCH-N-SKETCH allows the user to (i) select points of interest on the canvas and (ii) declare that the selected features should be related in some way.

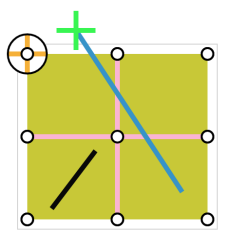

The screenshot on the left shows how features of the initial design can be selected. When clicking a positional feature, a crosshair is displayed that can be used to select position attributes of the point (see the top-left corner of the rectangle). Selected attributes are displayed in green (see the top endpoint of the top line).

To start adding relationships, let us make the top-left corner of the rectangle coincide with the endpoint of the top line (akin to "snapping" them together). By clicking the crosshairs of these two points, we indicate that both $\mathrm{x}$-positions and $\mathrm{y}$ positions, respectively, ought to be related. In order to specify our intended relationship, we click a button labeled Make Equal which instructs SKETCH-N-SKETCH to refactor the program so that, when re-evaluated, it results in the two point values being equal.

We repeat this selection and Make Equal process three more times, selecting the points at the bottom-left corner, the bottom-right corner, and the center of the logo.

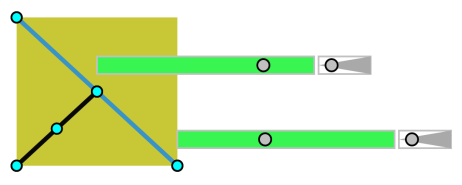

Sliders can also be toggled to relate attributes. By selecting the two color sliders (displayed in green when selected) and using Make Equal, and then selecting both line width sliders and using Make

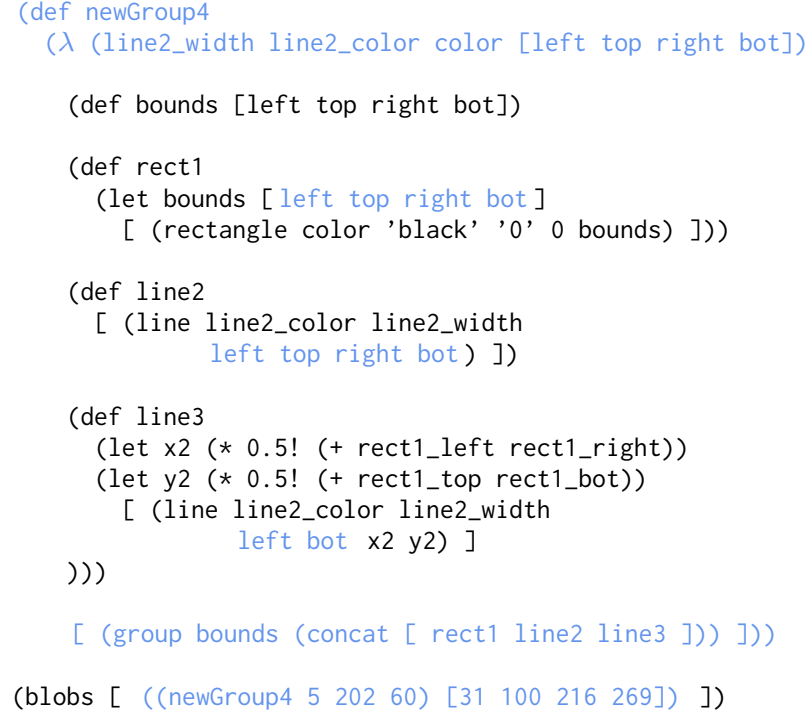

Figure 3. Overview example after grouping and abstracting shapes. The changes compared to Figure 2, made automatically by SKETCH-N-SKETCH, are highlighted in bolded blue.

Equal, SKETCH-N-SKETCH automatically transforms the original program (Figure 1) to the one shown in Figure 2 so that the width and color of both lines are equal. We will discuss the transformations in detail in the Tools for Relating Features section.

\section{Grouping Shapes}

Having built a program that implements the logo in terms of high-level design parameters (namely, the constants on lines 1, 2, 6, 9, and 10 in Figure 2), the final step is to make the design easy to reuse. To accomplish this, we select the three shapes, click Group to turn them into a single definition, and then click Abstract to turn the definition into a reusable function. Specifically, SKETCH-N-SKETCH refactors the three selected top-level definitions in the program into a single newGroup4 definition and adds function arguments for several design parameters. The three selected shapes are now generated by a single call to the newGroup4 function. Thus, the design is ready to be re-used. We will discuss these transformations (shown in Figure 3) in more detail in the Tools for Grouping Shapes section.

To recap, we were able to build the final program entirely using the new Draw, Relate, and Group tools, resulting in a high-level, readable function abstracted over the relevant design parameters. By leveraging the live synchronization inherited from previous work [9], we can easily change the design parameters - for example, to match the black-and-white configuration at the beginning of this section - by directly manipulating the logo and sliders.

\section{TOOLS FOR DRAWING SHAPES}

Having presented an overview of SKETCH-N-SKETCH, we now discuss the three new aspects of our work in more detail, starting with the Drawing tools in this section. Our choice to use general-purpose programs as the representation format 
poses two challenges: (1) the generated code should be readable so that it can be extended by the user if desired, and (2) the code should be structured in a way that facilitates the subsequent Relate and Group phases.

We have defined a library of stencil programs for drawing shapes that satisfy these two goals. In addition, we provide a way for converting user functions (either manually written or generated automatically by SKETCH-N-SKETCH transformations) into stencils that can be stamped out with direct manipulation. The screenshot on the right shows the Drawing toolbox in SKETCH-N-SKETCH.

\section{Program Structure}

A little program e can have arbitrary structure, but when e is of the form (def $x 1$ e1) ... (def $x n$ en) main, where main is of the form (blobs $\left[\mathrm{e} 1^{\prime} \ldots \mathrm{em}\right.$ ' $]$ ), we refer to the program structure as simple.

When drawing a new shape, simple program structure is preserved by adding the new shape definition (def y ey) to the end of the definition list and adding $y$ to the end of the blobs list (giving it the highest z-order). When a program e is not simple, we transform the program to (let y ey (addShapeToCanvas e y)), which refers to a simple library function for list concatenation.

\section{Stencils}

We have designed stencil programs for lines, rectangles, ovals, polygons, and paths (which comprise line and Bézier curve segments).

Figure 1 shows the stencils used to define rectangles and lines. The auto-generated code includes randomly chosen colors on lines 4,9 , and 14 for each shape, specified as integers between 0 and 500. The editor draws sliders next to each shape to control these colors, as well as the line width values on lines 9 and 14, with only minor differences compared to the previous version of SKETCH-N-SKETCH [9].

\section{Polygons}

To draw polygons, we use a stencil designed to make it easy to drag and stretch the entire shape (as one expects from a direct manipulation interface). For example, for the shape on the right, SKETCH-N-SKETCH generates the code below; the question marks can be ignored for now:

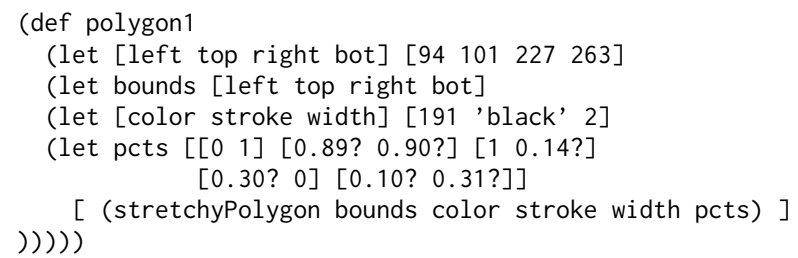

Notice that bounds defines the bounding box for the polygon, and each individual point is specified by a pair $[p q]$, where $\mathrm{p}$ (resp. q) is the x-position (resp. y-position) of the point relative to left and right (resp. top and bot). The little library function stretchyPolygon converts the percentages into absolute positions and then draws a raw SVG polygon. The function also draws a rectangle to surround the polygon.

The user can drag the bounding box to stretch all points accordingly (as shown on the right). Furthermore, we have designed the stretchyPolygon function so

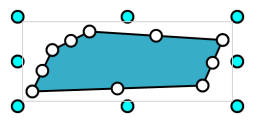
that dragging any of the points will affect the appropriate percentage values in the program.

For SVG paths, comprising straight line segments, quadratic Bézier curves, and cubic Bézier curves, we have designed and implemented a similar stencil called stretchyPath that allows an entire path to be stretched easily. More details can be found in our videos and Web demo.

\section{User-Defined Stencils}

The built-in stencils provide basic, scalable shapes. One of the benefits of using a programming language as the representation is that user-level designs can be treated on par with built-in primitives. In particular, a drawable shape may be described as any function that, given a bounding box argument, draws a shape within the bounding box. The built-in stencils satisfy this requirement, but so too can user-defined functions and ones automatically generated by SKETCH-N-SKETCH.

Recall the program in Figure 3, where the auto-generated newGroup4 function comprised the full logo design. Notice that the last argument to the function

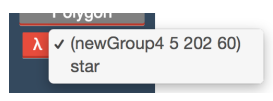
[left top right bot] denotes the bounding box. Our editor looks for such definitions in the program and adds them to a menu of user-defined stencils (depicted above).

Clicking the Lambda button then allows the user to draw a bounding box in the canvas, and then SKETCH-N-SKETCH adds a call to the selected function (i.e. lambda) with the bounding box argument. For example, we may create another instance of the logo using the Lambda tool, which adds a new function call to newGroup4 below. The arguments to each call serve as the design parameters for that instance.

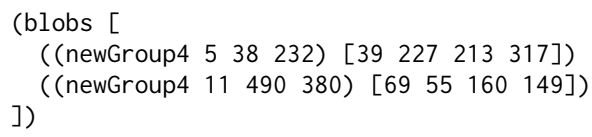

\section{TOOLS FOR RELATING ATTRIBUTES}

Once shapes have been drawn, the next step is to encode relationships in the program. There are several general concerns if the user were to manually edit the program: (1) identifying the relevant portions of the program (e.g. constants) that should be related; (2) boilerplate refactoring needed to move the relevant constants into the same part of the program; (3) explicitly programming the intended relationship; and (4) cleaning up the program after making the above changes.

SKETCH-N-SKETCH provides a four-step Relate workflow for these concerns. First, the user selects features of the output (via direct manipulation) that ought to be related. Second, 
SKETCH-N-SKETCH automatically refactors the program and digs a new hole expression where the selected features can be related in code. Third, the user or the system fills the hole with the desired relationship. Finally, SKETCH-N-SKETCH automatically cleans up unused and unnecessary definitions.

\section{Step 1: Select Features}

Each shape is defined by a set of attributes defined by the SVG specification [41]. For example, a rect includes $x, y$, width, height, and fill attribute values, and a line includes $\mathrm{x} 1, \mathrm{y} 1, \mathrm{x} 2, \mathrm{y} 2$, and stroke attribute values.

To select features, the editor displays clickable widgets that are used to toggle between selection and de-selection. For a positional feature, a crosshair is drawn which can be used to select the x-position (by clicking the vertical line through the crosshair), the y-position (the horizontal line), or both (the point in the center of the crosshair). For distance attributes like width, height, and radius, selection lines are drawn that span that distance on the

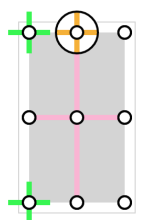
shape. Furthermore, as described in the Overview section, a slider can also be toggled (by clicking the backdrop of the slider) to select the value that it controls.

In addition to the primitive SVG features, the user may want to relate features that are derived in terms of the primitive ones, such as the bottom-right corner or the center of a rectangle. For each shape kind, our editor displays selection widgets for several common derived features. In the screenshot above, all of the crosshairs except the top-left corner of the rectangle identify derived features.

\section{Step 2: Dig Hole}

After the user selects features, pressing the Dig Hole button declares that they "should be related" in some way. For example, let us return to the initial program from the Overview that contains three unrelated shape definitions (Figure 1). The first relationship we added was between the top-left corner of the rectangle and the endpoint of the top line. After selecting these two points and clicking Dig Hole, SKETCH-N-SKETCH refactors part of the program as follows:

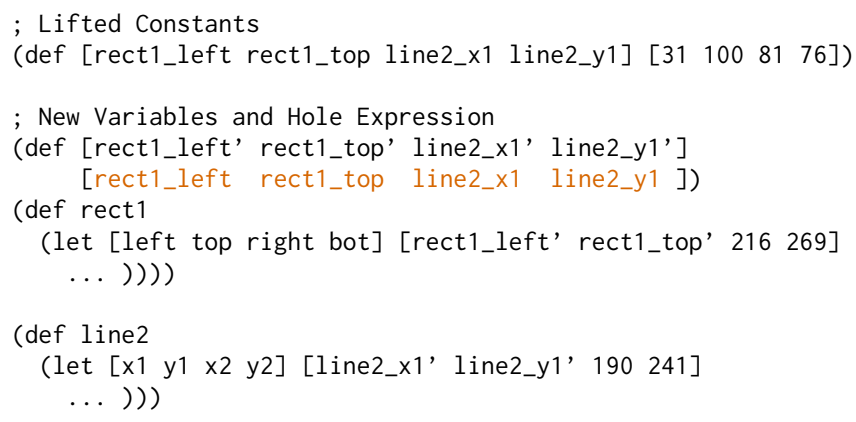

There are several aspects to notice about the transformed program. First, the constants that contribute to the selected features - the selected x-values (resp. y-values) are 31 and 81 (resp. 100 and 76) on lines 2 and 8 within the individual definitions - have been lifted into variables in the nearest common scope, in this case, before the rect 1 and line2 defini- tions. The names of new variables "collect" names from all of the scopes the constants have been lifted through. Second, a new variable has been defined for each lifted constant, suffixed with a prime. Third, the value of each primed variable is initialized to its previous value; we refer to the list expression that defines these primed variables as the hole expression, denoted in orange above. Finally, the primed variables are used wherever the original constants had been used before.

After this transformation, the program produces the same output values because the hole is filled with the previous constant values. The transformation does, however, create a single place where the intended relationship can be filled in.

\section{Names for Derived Features}

Primitive features correspond directly to expressions in the program text. Derived features, however, may not. If the user wishes to refer to the value of a derived feature when filling the hole, she would have to determine how to phrase the value in terms of what is mentioned in the program.

To simplify this process, we auto-generate named definitions for the derived features that are selected (in addition to the constant lifting). Recall that in the Overview section, we related the endpoint of the bottom line (a primitive feature defined by the $x 2$ and $y 2$ values on line 13 of the line 3 definition in Figure 1) and the center of the rectangle (a derived feature). To give explicit names for the latter feature, SKETCH-N-SKETCH inserts the following:

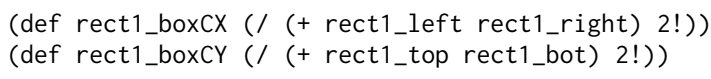

The user may then refer to these definitions if they are helpful for filling in the intended relationship. The exclamation points on the above constants are freeze annotations that instruct SKETCH-N-SKETCH not to change those constants during live synchronization [9].

\section{Step 3: Fill Hole}

Once Dig Hole has transformed the program, the next step is for the user to replace the default initial expression with the intended relationship. For example, when relating the topleft corner, we want to use a single constant to control both $\mathrm{x}$-values and another single constant to control both $\mathrm{y}$-values. Because the constants themselves do not matter much (they are easy to change via live synchronization), we might arbitrarily choose to edit the hole to use only rect1_left and rect1_top (leaving line2_x1 and line2_x2 unused):

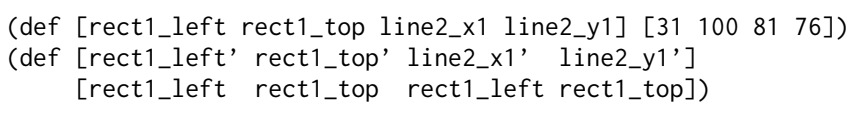

For all of the relationships in the Overview example, setting constants to be equal is the intended relationship. In general, however, the user may wish to code an arbitrary relationship.

\section{Step 4: Clean Up}

The Dig and Fill Hole operations introduce several new bindings, which are helpful during the filling process but may result in dead or unnecessary code afterwards. For the filled hole above, the line2_x1 and line2_y1 variables are unused, and the auto-generated rect1_left' and rect1_top' 
variables are unnecessary since they bind the same values as rect1_left and rect1_top. Furthermore, some (or most) of the named derived features may not be used. Therefore, we provide a Clean Up button to perform inlining, variable renaming, and other transformations to eliminate such expressions that result from the Select, Dig, and Fill workflow.

\section{Make Equal}

The Relate workflow assists in adding relationships to the program, but ultimately relies on the user to fill the hole. Ideally, this step could be automated for common cases.

Because the user often needs to make attributes equal (to "snap" positional features together and to make attributes like colors identical), our implementation provides automated support for filling the hole to encode equality. In particular, the Make Equal tool runs Dig Hole, attempts to fill the hole in a way that makes the selected features equal, and performs a Clean Up.

To fill the hole, we (i) consider the program expressions that produced the selected features, (ii) choose one program constant (i.e. degree of freedom) to remove from the program, and (iii) replace that constant with a variable that is defined in terms of the remaining constants.

\section{Solving Equations}

Our implementation employs and extends a prototype solver to reason about value-trace equations logged when evaluating a little program [9]. The details about the solver are beyond the scope of this paper. Below, we describe how it supports two common situations that arise in our examples. We write $\mathrm{n}^{\mathrm{x}}$ to denote that the constant literal $\mathrm{n}$ is bound to the variable $\mathrm{x}$ in a program.

One simple case is when relating two features that originate from constants. For example, to satisfy the equation

$$
31^{\text {rect1_left }}=81^{\text {line2_x1 }}
$$

we arbitrarily choose a constant to eliminate, say, line2_x1, and replace all occurrences of line2_x1 with rect1_left.

Features are often defined by more complicated expressions than just constants. Suppose we want to align the bottom edge of the stretchy polygon 1 from before. Selecting the $y$-positions of the bottom-left and bottom-right corners (highlighted in green in the adjacent

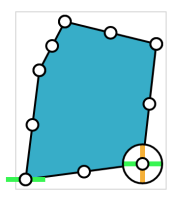
screenshot) and pressing Make Equal leads to the following equation:

$$
263^{\text {bot }}=\left(+101^{\text {top }}\left(* 0.90\left(-263^{\text {bot }} 101^{\text {top }}\right)\right)\right)
$$

There are three possible degrees of freedom (i.e. constants) to remove from the program: the values of top, bot, and the percentage 0.90 . Arbitrarily choosing to remove top or bot would break the structure of the design. In this situation, the intent of the original program was that the same program expression defines all points, and only the percentages varied. Thus, we would like to only remove the percentage constants, not the bounding box ones.
To help disambiguate this common case, a number in the program annotated with a question mark, written $n$ ?, serves as a hint to the solver that it should prefer to remove this constant when given a choice. Notice that the percentages in polygon 1 have ? annotations whereas as the bounds constants do not. We designed the polygon stencil this way so that running Make Equal on a point inside the bounding box will change only the position of the point, not the proportions or position of the entire shape. For the equation above, the constant 0.90 is removed from the program and replaced with a variable that binds 1.00 , the position ratio for the bottom of the bounding box:

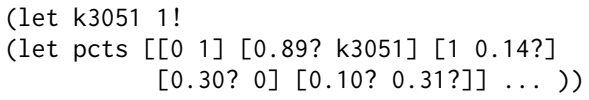

Thus, the bottom-right corner is snapped to the bottom of the bounding box. Repeating this Make Equal process for each remaining side produces the "snip polygon" shown above.

\section{TOOLS FOR GROUPING SHAPES}

Once the relationships in a program have been structured around a set of design parameters, the last step is to refactor the program so that it can be reused easily. In the Group workflow, SKETCH-N-SKETCH provides several program transformation tools for different abstraction patterns, described below.

\section{Group}

The Group tool performs three steps. First, a new top-level definition is created consisting of the selected blobs (blobs are either names of top-level definitions or calls to top-level functions). Second, a new bounding box [left top right bot] is computed to span the bounding boxes of the selected blobs. Lastly, so that the entire group scales proportionally when stretched, the bounding box of each selected blob is rewritten as a percentage in terms of the new one; this approach is similar to that used by stretchyPolygon.

In Figure 3, notice how the newGroup4 definition contains the three shape definitions that had previously been at the top-level (in Figure 2). To rewrite the bounding box of rect1 in terms of the new group bounding box [left top right bot], the Group transformation would usually generate the following:

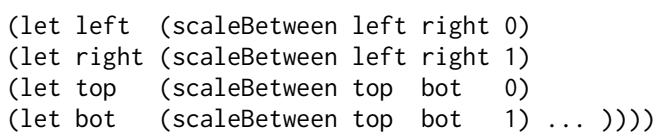

But when the relative percentages are equal to 0 or 1 , SKETCH-N-SKETCH instead generates the equivalent but simpler definition on line 7 of Figure 3. In the Evaluation section, we will discuss an example where the hierarchy of bounding boxes is more complicated.

\section{Abstract}

The Group tool turns multiple top-level definitions (or calls to top-level definitions) into a single definition. Next, the Abstract tool turns a top-level definition into a function that 
is abstracted over several parameters. The challenge for automation is choosing which free variables and constant literals from the body of the definition to abstract.

To facilitate common use in SKETCH-N-SKETCH, our heuristic is to abstract over (non-frozen) constants and, furthermore, those that have been assigned a name. Using this approach, SKETCH-N-SKETCH introduces variables for seven design parameters on line 2 of Figure 3.

In addition to abstracting such constants, the transformation also syntactically looks for the bounding box pattern and, when present, makes a single bounds parameter. For example, notice how the four bounds parameters are put into a single list argument and made the last parameter on line 2 of Figure 3. With this structure, the Lambda tool recognizes that it is a new stencil that can be stamped out via the Draw toolbox. With the new abstraction, the selected shapes are now generated by a single call to the newGroup4 function (line 23).

\section{Duplicate and Merge}

In addition to Abstract, which turns a single blob into a reusable function, SKETCH-N-SKETCH supports a second workflow for abstraction. First, the Duplicate tool copies the code for the selected blob verbatim. As such, manipulating one copy does not affect the other.

Next, after making changes to some attributes, the user may select the copies and use the Merge tool. The editor syntactically compares the definitions for equivalence modulo leaves of the AST (i.e. constants). If the definitions are structurally equivalent, the program is transformed to turn the separate definitions into a single function that is abstracted over any constants for which not all copies agree.

By using Duplicate and then Merge, the user is, in effect, explicitly specifying the differences (via the direct manipulation changes) between the copies, and all other attributes are implicitly encoded to be equal because they do not become function parameters. Manipulating any such attribute in one copy will then affect all copies. We will discuss examples in the Evaluation section that use this pattern.

\section{IMPLEMENTATION}

We have extended SKETCH-N-SKETCH [9] with tools for the Draw, Relate, and Group workflows. The extended system is written in more than 13,000 lines of Elm [11] and JavaScript code (approximately half of which consists of our extensions) and runs as a standalone Web application. Figure 4 shows a screenshot of our editor, which displays a program and its output side-by-side with several tools in between for editing the code and the canvas.

\section{Code Formatting}

Maintaining the readability of the program is one of our design objectives. Therefore, our implementation takes care to generate and transform readable code. The code listings throughout the paper are identical to the ones generated and transformed by our implementation, except for minor whitespace changes to improve readability and to fix a couple of instances where our current implementation inserts one too many or one too few spaces or lines breaks.

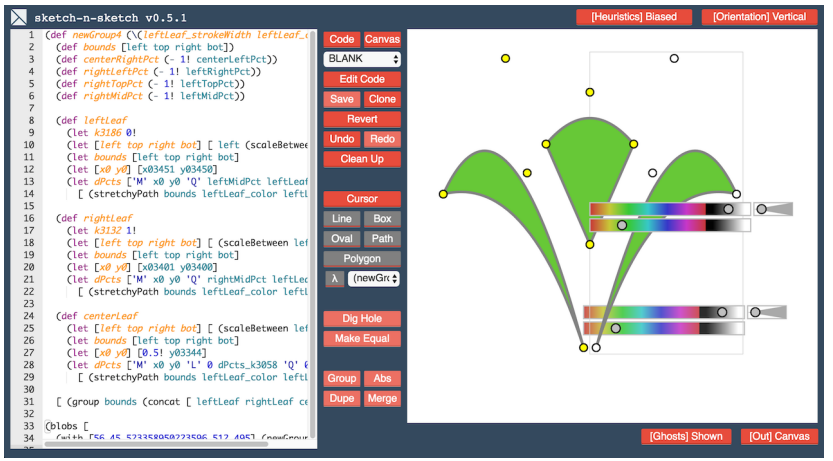

Figure 4. Screenshot of SKETCH-N-SKETCH V0.5.1

\section{EVALUATION}

To evaluate our system, we have implemented three example designs, in addition to the one described in the Overview section. We also discuss examples of how using a generalpurpose programming language offers opportunities for integrating custom user libraries with direct manipulation tools. To demonstrate the interactions, we have recorded several videos and posted them on the Web.

\section{Examples}

In the Overview section, we described how to build one example program from scratch. Below, we discuss three more.

\section{SKETCH-N-SKETCH Logo Revisited}

The logo described in the Overview consisted of two lines drawn over a rectangle. That construction is insufficient if we want to use different colors for the three parts or if we want the negative space to be truly transparent. Instead, we can construct the logo with three polygons.

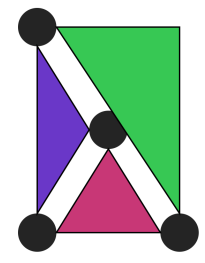

To align and properly space the polygons, we draw four identical helper circles to act as spacers. We Merge the helper circles to ensure their radii are identical. Then, we use Make Equal to align each corner of each polygon to the edge of an adjacent helper circle. To make the design rectangular, we align adjacent corners with Make Equal. Similarly, we center the central helper circle by aligning it to a midpoint of each side. The design so far is shown on the left.

To equalize the width and height of the design, we draw a large helper circle (not shown above) and use Make Equal four times to align its bounding box with that of the logo.

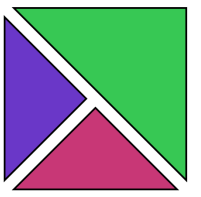

To hide the helper circles from the final output, we could wrap each helper shape in the code with a call to a library function called ghost, which would allow us to hide or show them via a button in the interface. Alternatively, since we are done adding relationships to our design, we simply delete the helper shapes. After doing so, we discover that all our constraints are preserved. We can then adjust the spacing between the polygons as well as resize the entire shape using live synchronization. 
The entire design is constructed using the drawing tools, 18 uses of Make Equal, and a single code edit to remove the helper shapes. However, there is a cost to this ease. The repeated applications of Make Equal pollute the top level of our code. After four design parameters that specify the size of the logo and the width of the gap, there are 12 lines of mathematically correct but unintelligible equations:

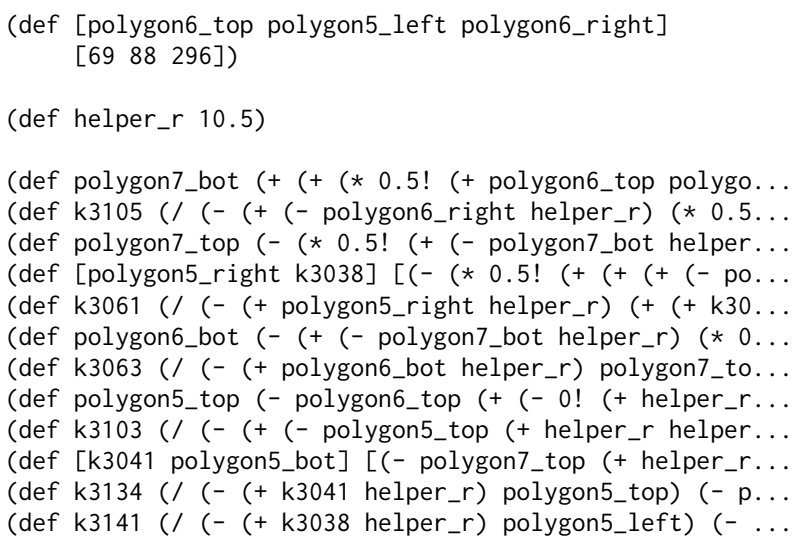

In situations where readability is a priority but where SKETCH-N-SKETCH generates expressions like the above, one could fall back on Dig Hole instead of Make Equal and program the relationships manually. However, we believe the automatically generated code can be significantly improved in future work by incorporating a smarter algebraic simplifier; our current implementation supports only a few local syntactic transformations.

\section{Garden Logo}

Our next example borrows from the logo of the Chicago Botanic Garden (www. chicagobotanic.org), which consists of three leaves symmetric across a vertical axis. We start by drawing three Bézier curves, and then manually edit the code to rename the auto-generated names to leftLeaf, rightleaf, and centerLeaf. We equate the color, strokeColor, and strokeWidth attributes with several

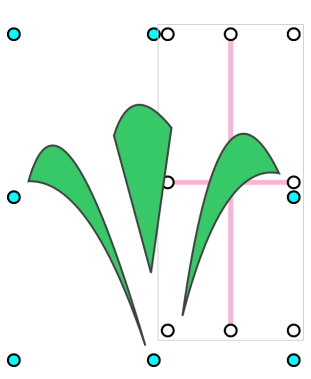
Make Equal operations.

Next, we Group the three leaves, which transforms the program as follows:

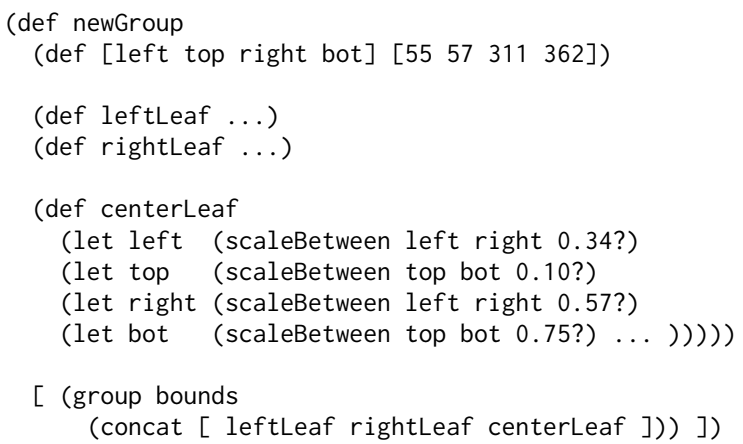

Notice how the bounding box for the group comprises the bounding boxes for the three leaves. As described in the Tools for Grouping Shapes section, the bounding boxes for the constituent shapes are rewritten in terms of the new one. For example, the new bounding box for centerLeaf is specified using percentages; these can be changed by directly manipulating the box drawn around centerLeaf.

Our final step is to encode horizontal alignment and vertical symmetry. For horizontal alignment, there are five pairs of y-positions (specified as percentages) to relate, each of which can be handled by Make Equal. For vertical symmetry, there are five pairs of $\mathrm{x}$-positions (also specified as percentages) to relate. For each pair, we use Dig Hole to identify two percentages $p$ and $q$ and edit the hole to replace the auto-generated primed variable q' with $(-1.0 ! p)$, which encodes symmetry across the central axis. Lastly, we identify the two percentages in the program that control the x-positions of the top and bottom of the centerLeaf - when hovering over features, SKETCH-N-SKETCH highlights the relevant constants in the code - and edit them to be 0.50 ! so that centerLeaf always remains in the center. One configuration of the final design is depicted in Figure 4.

Just as Make Equal lets the user "Align" shapes without manual code edits, in future work it would be useful to add fillers to automatically "Reflect" and "Distribute" shapes.

\section{Coffee Mug}

Our last example is a steaming mug of coffee. We start by drawing two ellipses for the handle and a rectangle for the body. We select the edges of the inner and outer ellipses, use Dig Hole to lift their values, and edit the program to center the inner ellipse inside the outer, but 0.20 ! times smaller. To affix the handle to the side of the mug, we select the center of the el-

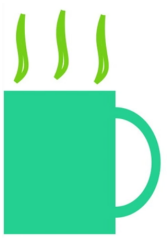
lipses and the midpoint of the rectangle's right edge, and align them with Make Equal. We also use Make Equal to match the handle color to the body color. For the steam, we draw one path, use Duplicate to make two copies, reposition the copies, and use Merge to re-combine their definitions in the program.

\section{Ul and Library Co-Design}

The Draw toolbox provides a set of "built-in" shapes, but the stencil code behind them call ordinary little functions - rectangle, line, oval, stretchyPolygon, and stretchyPath - which happen to be defined in the standard library. The Lambda tool is a way to integrate user (or library) customization with native features. We describe two additional opportunities for future work below.

\section{Custom Scaling}

The "stretchy" semantics provided by stretchyPolygon is useful in many situations but is not the only scaling semantics the user may want for a particular shape. For example, recall the snip polygon shown in the Tools for Relating Features section. A different reasonable intention is for the absolute distance between the snip and the bounding box to remain fixed, even as the polygon is scaled. 
Our library provides a stickyPolygon function that provides this interpretation of scaling, where points "stick" to the nearest corner. To draw the same polygon described by polygon1 in the Tools for Drawing Shapes section, the following code uses absolute offsets, rather than percentages, to describe

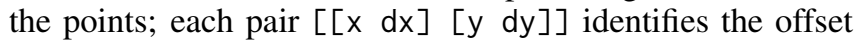
$\left[\begin{array}{ll}d x & d y\end{array}\right]$ from a particular corner $\left[\begin{array}{cc}x & y\end{array}\right]$ :

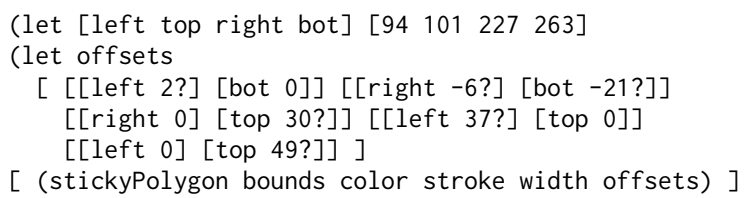

An even different scaling behavior commonly found in existing tools combines both of the above; features, such as rounded or snipped corners, may stretch up to a certain point after which they stick at a fixed distance. Because little is a general-purpose language, we can write a stretchySnipPolygon function to encode this behavior as a small variation on the previous functions.

\section{Custom Features}

For each kind of shape, SKETCH-N-SKETCH draws selection widgets for a set of derived features. These features are currently hard-coded in our implementation, but there might be other features relevant for a particular design. In the adjacent screenshot, we modified our program to draw a helper dot to identify the maximum snip distance of a stretchySnipPolygon. It may be useful to allow users and libraries to customize the derived features displayed and manipulated.

\section{DISCUSSION}

To wrap up, we compare SKETCH-N-SKETCH to related work, augmenting the landscape described in the Introduction. We also discuss limitations of our approach and opportunities for future work.

\section{Combining Programming and Direct Manipulation}

Programmatic and direct manipulation have been combined in a myriad of diverse configurations. Prior systems have generally emphasized direct manipulation over programming, with the underlying programs expressed in domain-specific languages or with special data structures.

\section{Scriptable Direct Manipulation Editors}

Some direct manipulation systems provide scripting APIs that allow users to run editor commands programmatically. To ease the programming burden, some editors allow the user to record their actions into a macro script (e.g. SolidWorks [12]) or echo equivalent scripting commands for every action to facilitate copy-paste scripting (e.g. Maya [2]).

\section{Parametric Computer-Aided Design}

In parametric feature-based $C A D$ systems, user actions are concatenated into a procedure (i.e. script), hidden from the user, that specifies the step-by-step creation of the design. Features of new elements can be defined in terms of the parameters of existing elements. When the user changes a parameter, all commands in the script that depend on it are automatically re-run - for example, if the user resizes the main cylinder of a screw, the screw head defined to be $1.5 \mathrm{x}$ wider will automatically be resized as well [14]. Grouping and simple repetition are also generally supported, and the EBP system [29] additionally implemented programming by demonstration interactions to specify loops and conditionals.

\section{Procedural Modeling}

In procedural modeling systems, specialized algorithms automatically generate complex 2D or 3D content such as trees, terrain, or road networks. Some of these systems allow algorithmic parameters to be adjusted by directly manipulating the output. However, the algorithms themselves are usually built-in to the system and cannot be altered by the user [35].

Algorithms based on generative shape grammars generally allow text-based grammar modifications. Richer direct manipulation interactions have also been explored to change individual rule parameters $[19,24]$, to copy-paste rules [4], to manipulate grammars visually $[34,28,24]$, and to infer grammars from sample output $[1,39,5]$.

\section{Programming by Example or Demonstration}

Many PBE [23] and PBD [10] systems for graphics editing support operations analogous to the drawing, relating, and grouping of this paper but use domain-specific representations for the underlying program (e.g. [20, 25, 22]). Two recent systems are QuickDraw, which infers both constraints [8] and procedural repetition [7] based on a userprovided sketch, and Drawing Dynamic Visualizations [38], in which direct manipulation operations construct a program as a graphical history that supports constraints, looping, and parameterized abstraction.

\section{Our Approach}

In contrast, we start with a general-purpose language and codify the user's actions by transforming the program. These two features - a general-purpose language and code transformations - are the key differentiators of our approach; all of the above systems either rely upon a domain-specific language or provide only basic features for editing the code.

\section{Live Synchronization}

Our previous version of SKETCH-N-SKETCH required that the initial program be written using traditional text-based editing, a burden reduced by our new techniques.

Once written, live synchronization [9] allowed changes to the output of a program to immediately change appropriate constants in the program. To do so, SKETCH-N-SKETCH records run time-traces that relate the input program to its output; these relationships form value-trace equations. To synthesize program updates, the system attempts to solve one valuetrace equation per updated attribute by changing the value of exactly one constant in the program. When there are ambiguities (i.e. multiple constants to change), SKETCH-N-SKETCH uses heuristics to automatically choose without asking the user for help.

When extending SKETCH-N-SKETCH, we inherited the live synchronization approach - along with the limitations that stem from the one-equation, one-constant design - with only 
minor changes. One modification was to add a new BOX primitive specified by left, right, top, and bot values which translates to the SVG rect primitive.

There are two ways in which our new features are designed with the behavior of live synchronization in mind. First, we carefully designed our scalable polygon and path stencils so that they interact well with live synchronization; directly manipulating the exterior points also manipulates the bounding box, and directly manipulating the interior points manipulates the appropriate constants when running SKETCH-N-SKETCH in "biased" heuristics mode [9].

Second, the ways that our Group operations transform the program favor an approach where bounding boxes are defined with constants in the program and inner shapes are defined relative to the bounding box. Such programs lend themselves to more intuitive live synchronization than ones where shape attributes are defined directly and bounding boxes are implicitly derived from them.

Nevertheless, the ideas behind our shape stencils and program transformations could be re-purposed to favor other programming patterns instead.

\section{Constraint-Oriented Programming}

Constraint-oriented programming systems, including SketchPad [36] and ThingLab [6] among others [30, 13, 27], allow users to specify declarative relationships that augment procedural programs. Constraint solvers (e.g. [3]) attempt to satisfy the declared relationships.

Although our Relate workflow suggests the declarative feel of constraints, our representation format is a concrete, deterministic program. Since we do not rely on additional constraint or solver state, our functional programs may be more easily reused in other, general-purpose programming domains.

Several user interaction techniques have been explored for defining and breaking constraints [17, 15, 21], some of which might be applied to our system to improve our UI for declaring relationships.

\section{Automated Refactoring}

Some of our program transformations - Clean Up, Group, Abstract, and Merge - can be viewed as variations on common program refactorings, whereas some - Dig Hole - do not have direct analogs.

Our Dig Hole approach lifts all relevant constants in the program into a common scope. An alternative design might look for additional expressions (beyond just constants) where an intended relationship might be filled in.

Currently, our Group transformations work only for programs in the simple structure described earlier. For cases where the program has more complicated structure, and for abstracting finer-grained pieces of code than just top-level definitions, it would be useful to develop a more general methodology for syntactic abstraction of source programs, probably taking into account run-time traces [9]. Such generalized approaches might benefit from interactive, visual editors such as those described below.

\section{Structured and Visual Editors}

It may be useful in future work to design a more visual, structured editor $[37,16]$ — particularly one like Barista [18] that also supports unstructured, text-based editing — specifically for the workflow that arises in SKETCH-N-SKETCH.

For example, because our tools automatically generate and transform code, the user may often want to easily rename and reorder definitions. In addition, whereas our current Merge and Abstract transformations use specific design heuristics to decide what parts of a program expression to abstract, it would be useful to provide a way for the user to interact with the system during the process. Finally, although the named definitions for derived features inserted by Dig Hole are useful, it would be better to provide a visual connection between the rendered features and the generated program expressions.

In general, such improvements may be lead to new direct manipulation features for code editing itself. Widgets [9] and so-called "scrubbing tools" $[38,26]$ can be viewed as examples of this notion.

\section{Conclusion}

The new drawing, relating, and grouping interactions we added to SKETCH-N-SKETCH are potentially useful enhancements for users that currently choose languages (e.g. Processing) and libraries (e.g. D3) to programmatically generate 2D vector graphics.

In the longer-term, we view this work as a milestone towards a vision where (a) experts may choose to use tools that mix programming and direct manipulation in a variety of domains, and where (b) novices get some of the benefits of programming by (i) having expert library writers customize tools with new purely GUI-based features, (ii) learning a bit of programming through the live connection and semi-automated programming tools, and (iii) applying and extending programming-by-example to these settings. In pursuit of this vision, we will continue to explore how rich direct manipulation capabilities can provide interactive and intuitive environments for general-purpose programming languages. 


\section{REFERENCES}

1. Aliaga, D. G., Rosen, P. A., and Bekins, D. R. Style Grammars for Interactive Visualization of Architecture. IEEE Transactions on Visualization and Computer Graphics (TVCG) (2007).

2. Autodesk Inc. Maya. http: //www. autodesk. com/products/maya/overview.

3. Badros, G. J., Borning, A., and Stuckey, P. J. The Cassowary Linear Arithmetic Constraint Solving Algorithm. Transactions on Computer-Human Interaction (TOCHI) (2001).

4. Barroso, S., Besuievsky, G., and Patow, G. Visual Copy $\&$ Paste for Procedurally Modeled Buildings by Ruleset Rewriting. Computers \& Graphics (2013).

5. Bokeloh, M., Wand, M., and Seidel, H.-P. A Connection Between Partial Symmetry and Inverse Procedural Modeling. Transactions on Graphics (TOG) (2010).

6. Borning, A. The Programming Language Aspects of ThingLab. Transactions on Programming Languages and Systems (TOPLAS) (October 1981).

7. Cheema, S., Buchanan, S., Gulwani, S., and LaViola, Jr., J. J. A Practical Framework for Constructing Structured Drawings. In International Conference on Intelligent User Interfaces (IUI) (2014).

8. Cheema, S., Gulwani, S., and LaViola, J. QuickDraw: Improving Drawing Experience for Geometric Diagrams. In Conference on Human Factors in Computing Systems (CHI) (2012).

9. Chugh, R., Hempel, B., Spradlin, M., and Albers, J. Programmatic and Direct Manipulation, Together at Last. In Programming Language Design and Implementation (PLDI) (2016).

10. Cypher, A. Watch What I Do: Programming by Demonstration. MIT Press, 1993.

11. Czaplicki, Evan. Elm. http://elm-lang.org.

12. Dassault Systèmes SOLIDWORKS Corp. SOLIDWORKS Macros. http://help. solidworks.com/ 2016/English/api/sldworksapiprogguide/GettingStarted/ SolidWorks_Macros.htm.

13. Felgentreff, T., Millstein, T., Borning, A., and Hirschfeld, R. Checks and Balances: Constraint Solving Without Surprises in Object-Constraint Programming Languages. In Object-Oriented Programming, Systems, Languages, and Applications (OOPSLA) (2015).

14. Girard, P. Bringing Programming by Demonstration to CAD Users. In Your Wish is My Command: Programming by Example. Morgan Kaufmann Publishers Inc., 2001.

15. Gleicher, M., and Witkin, A. Drawing with Constraints. The Visual Computer: International Journal of Computer Graphics (1994).

16. Habermann, A. N., and Notkin, D. Gandalf: Software Development Environments. IEEE Transactions on Software Engineering (TSE) (1986).
17. Hottelier, T., Bodik, R., and Ryokai, K. Programming by Manipulation for Layout. In Symposium on User Interface Software and Technology (UIST) (2014).

18. Ko, A. J., and Myers, B. A. Barista: An Implementation Framework for Enabling New Tools, Interaction Techniques and Views in Code Editors. In Human Factors in Computing Systems (CHI) (2006).

19. Krecklau, L., and Kobbelt, L. Interactive Modeling by Procedural High-Level Primitives. Computers \& Graphics (2012).

20. Kurlander, D. Graphical Editing by Example. $\mathrm{PhD}$ thesis, Columbia University, 1993.

21. Kurlander, D., and Feiner, S. Inferring Constraints from Multiple Snapshots. Transactions on Graphics (TOG) (1993).

22. Lieberman, H. Mondrian: A Teachable Graphical Editor. In Watch What I Do: Programming by Demonstration. MIT Press, 1993.

23. Lieberman, H. Your Wish is My Command: Programming by Example. Morgan Kaufmann Publishers Inc., 2001.

24. Lipp, M., Wonka, P., and Wimmer, M. Interactive Visual Editing of Grammars for Procedural Architecture. Transactions on Graphics (TOG) (2008).

25. Maulsby, D. L., Witten, I. H., and Kittlitz, K. A. Metamouse: Specifying Graphical Procedures by Example. In Conference on Computer Graphics and Interactive Techniques (SIGGRAPH) (1989).

26. McDirmid, Sean. A Live Programming Experience. Future Programming Workshop, StrangeLoop 2015. https: //www. youtube. com/watch?v=YLrdhFEAiqo.

27. Nelson, G. Juno, A Constraint-Based Graphics System. In Conference on Computer Graphics and Interactive Techniques (SIGGRAPH) (1985).

28. Patow, G. User-Friendly Graph Editing for Procedural Modeling of Buildings. IEEE Computer Graphics and Applications (2012).

29. Pierra, G., Potier, J.-C., and Girard, P. The EBP System: Example Based Programming System for Parametric Design. In Modelling and Graphics in Science and Technology. Springer Berlin Heidelberg, 1996.

30. Samimi, Hesam and Warth, Alex. Sketchpad14. http://www. cdglabs. org/sketchpad14.

31. Schachman, Toby. Apparatus. http://aprt.us/.

32. Schuster, C., and Flanagan, C. Live Programming by Example: Using Direct Manipulation for Live Program Synthesis. In LIVE Workshop (2016).

33. Shneiderman, B. Direct Manipulation: A Step Beyond Programming Languages. Computer (August 1983). 
34. Silva, P. B., Müller, P., Bidarra, R., and Coelho, A. Node-Based Shape Grammar Representation and Editing. In Workshop on Procedural Content Generation in Games (2013).

35. Smelik, R. M., Tutenel, T., Bidarra, R., and Benes, B. A Survey on Procedural Modelling for Virtual Worlds. Computer Graphics Forum (2014).

36. Sutherland, I. Sketchpad, A Man-Machine Graphical Communication System. PhD thesis, MIT, 1963.

37. Teitelbaum, T., and Reps, T. The Cornell Program Synthesizer: A Syntax-Directed Programming Environment. Communications of the ACM (1981).

38. Victor, Bret. Drawing Dynamic Visualizations. http: //worrydream. com/\#!/DrawingDynamicVisualizationsTalk.
39. Št'ava, O., Beneš, B., Měch, R., Aliaga, D. G., and Krištof, P. Inverse Procedural Modeling by Automatic Generation of L-systems. Computer Graphics Forum (2010).

40. Wang, X., Zhang, L., Xie, T., Xiong, Y., and Mei, H. Automating Presentation Changes in Dynamic Web Applications via Collaborative Hybrid Analysis. In International Symposium on the Foundations of Software Engineering (FSE) (2012).

41. World Wide Web Consortium (W3C). Scalable Vector Graphics (SVG) 1.1 (Second Edition). http://www.w3.org/TR/SVG11/. 